\title{
Bloqueio AV total congênito: novo modelo experimental para avaliação do marcapasso fetal
}

\author{
Renato S. ASSAD*, Marcelo B. JATENE* ${ }^{\star}$, Luiz Felipe P. MOREIRA*, Paulo C. SALES ${ }^{*}$, Vera Demarqui \\ AIELLO*, Roberto COSTA*, Frank L. HANLEY ${ }^{\star}$, Adib D. JATENE*
}

\begin{abstract}
ASSAD, R. S.; JATENE, M. B.; MOREIRA, L. F. P.; SALES, P. C.; AIELLO, V. D.; COSTA, R.; HANLEY, F. L.; JATENE, A. D. - Bloqueio AV total congênito: novo modelo experimental para avaliação do marcapasso fetal. Rev. Bras. Cir. Cardiovasc., 9 (3): 133-140, 1994.

RESUMO: O implante de marcapasso epicárdico em fetos via toracotomia é um procedimento potencialmente mais seguro e eficaz para se tratar o bloqueio AV total congênito (BAVT), quando associado à hidropsia fetal e refratário ao tratamento clínico. Este estudo foi desenvolvido com o objetivo de avaliar as características eletrofisiológicas de dois eletrodos epicárdicos através de novo modelo experimental de BAVT congênito induzido pela crioablação do nó AV. Foram aplicados, em 2 grupos de 6 fetos de ovelhas ( $80 \%$ da gestação), um eletrodo de rosqueamento (1,5 voltas) e outro de sutura epicárdica. O BAVT foi obtido em todos os fetos, não sendo observado nenhum rítmo de escape ventricular. Os limiares de estimulação foram baixos para ambos os eletrodos, com valores inferiores para o eletrodo de rosqueamento com largura de pulso abaixo de 0,9 mseg $(p<0,03)$. A corrente medida no limiar de voltagem com largura de pulso abaixo de $0,5 \mathrm{mseg}$ foi menor para o eletrodo de rosqueamento $(p<0,048)$. A resistência dos 2 eletrodos medida com voltagem constante não foi estatisticamente diferente $(441,8 \pm 13,7 \Omega$ para o eletrodo de rosqueamento versus $480,2 \pm$ $59,2 \Omega$ para o eletrodo de sutura epicárdica). Não houve diferença estatisticamente significante $(p>0,20)$ na amplitude da onda $R$ dos 2 eletrodos. $O$ slew rate foi significativamente maior para 0 grupo de fetos com eletrodo de rosqueamento $(1,40 \pm 0,2$ versus $0,62 \pm 0,2 \mathrm{~V} / \mathrm{seg} . p=0,04)$. O método é simples e reprodutível para avaliação do marcapasso fetal, sendo que o eletrodo de rosqueamento representa a melhor opção, quando houver indicação de implante de marcapasso em fetos.
\end{abstract} fetal.

DESCRITORES: cardiologia fetal; bloqueio AV, congênito; cirurgia fetal; arritmias fetais; marcapasso

\section{INTRODUÇÃO}

A bradiarritimia ocasionada pelo bloqueio AV total congênito (BAVT) ocorre em $4 \%$ a $15 \%$ dos casos de arritimia fetal ${ }^{15,22}$. Embora o BAVT possa gerar uma freqüência cardíaca de $30 \%$ a $50 \%$ do rítmo fetal normal, este distúrbio do rítmo fetal é bem tolerado na ausência de anomalias sistêmicas, cardíacas ou de insuficiência placentária. Entretanto, o prognóstico é muito ruim quando o BAVT se associa à hidropsia fetal como conseqüência de insuficiência cardíaca freqüência dependente ${ }^{14,15}$. Um estudo multicêntrico sobre história natural desta afeç̧ão demonstrou que a hidropsia fetal ocorreu em $22(40 \%)$ dos 55 fetos com o diagnóstico de BAVT congênito ${ }^{23}$. Esta associação mórbida de doenças apresentou êxito letal em todos os casos, independentemente da presença de cardiopatia congênita associada.

Trabalho realizado na Divisāo de Pesquisa do Instituto do Coração do Hospital das Clínicas da Faculdade de Medicina da Universidade de São Paulo, com o apoio da Fundação E. J. Zerbini (Projeto n² 71191/26). São Paulo, SP, Brasil.

Recebido para publicaçăo em setembro de 1994.

- Do Instituto do Coraçāo do Hospital das Clínicas da Faculdade de Medicina da Universidade de São Paulo.

* Da Universidade da Califórnia. São Francisco, EUA.

Endereço para correspondência: Renato Assad. Av. Dr. Eneas de Carvalho Aguiar, 44. Divisão Cirúrgica. CEP 05403-100, São Paulo, SP, Brasil. 
ASSAD, R. S.; JATENE, M. B.; MOREIRA, L. F. P.; SALES, P. C.; AIELLO, V. D.; COSTA, R.; HANLEY, F. L.; JATENE, A. D. Bloqueio AV total congênito: novo modelo experimental para avaliação do marcapasso fetal. Rev. Bras. Cir. Cardiovasc., 9 (3): $133-140,1994$.

Caso o baixo débito e/ou freqüência cardíaca não possam ser revertidos com o tratamento clínico através de drogas cronotrópicas positivas ou esteróides $5,9,18,23$, o implante de marcapasso em fetos deve ser o próximo passo no tratamento do BAVT congênito.

CARPENTER et alii ${ }^{6}$ relataram um implante de marcapasso ventricular em feto humano para tratamento de BAVT congênito, aparentemente sem trauma materno-fetal. Entretanto, a abordagem transuterina e transtorácica de implante do eletrodo apresenta algumas desvantagens, tais como a possibilidade de deslocamento do eletrodo ou lesão fetal com a movimentação do feto. Além do mais, esta abordagem requer repouso materno e estaria sujeita à corio-amnionite infecciosa.

Com os avanços dos métodos diagnósticos e o maior domínio da história natural do BAVT congênito $1,2,10,16,20,23$, bem como a experiência inicial da cirurgia fetal em humanos 17 , o implante do marcapasso epicárdico fetal via toracotomia pode ser um procedimento potencialmente mais seguro e eficaz.

A avaliação eletrofisiológica durante o implante é fundamental para assegurar o comando do marcapasso durante a gestação, devendo ser profundamente analisado em modelo experimental. A sensibilidade e o estímulo representam funções distintas de um gerador de marcapasso de demanda e devem ser testados separadamente. Apenas um modelo animal de BAVT foi publicado ${ }^{8}$. Entretanto, o índice de $90 \%$ de BAVT obtido com a técnica descrita foi difícil de se reproduzir em nossos laboratórios. No presente estudo, foi desenvolvido um novo modelo experimental de BAVT fetal para avaliar e comparar os parâmetros eletrofisiológicos de dois eletrodos epicárdicos distintos, sem a influência da alta freqüência cardíaca fetal.

\section{CASUÍSTICA E MÉTODOS}

\section{Anestesia}

Doze fetos de ovelhas com idade gestacional entre 110 e 115 dias $(75 \%$ a $80 \%$ da gestação, termo $=147$ dias) foram estudados. Esta fase da gestação corresponde a um feto humano entre 28 e 32 semanas, com massa cardíaca quase igual à de feto ovino, entre $6,2 \mathrm{~g}$ a $14,0 \mathrm{~g}^{24}$. O eixo longitudinal do ventrículo de fetos ovinos é de aproximadamente $25 \mathrm{~mm}$ nesse período gestacional. De acordo com a avaliação ultra-sonográfica da geometria cardíaca fetal humana feita por WLADIMIROFF et alii 27,28 em idade gestacional equiva- lente, o eixo transverso varia entre $11,2 \mathrm{~mm}$ a $13,1 \mathrm{~mm}$, enquanto o eixo longitudinal é também quase igual ao de fetos de ovinos $(22,0 \mathrm{~mm} \pm 2,3 \mathrm{~mm})$.

As ovelhas receberam como profilaxia antibiótica cefalotina $1 \mathrm{~g}$ e gentamicina $40 \mathrm{mg}$ por via intramuscular (I.M.) a cada 12 horas, durante três dias, com início logo antes da operação. A anestesia materna foi induzida com ketamina $(10 \mathrm{mg} / \mathrm{kg}$ ) I.M. e mantida com infusão peridural de marcaína $(1 \mathrm{mg} /$ $\mathrm{kg}$ ). A sedação foi feita de acordo com as necessidades (pentobarbital $100 \mathrm{mg}$ IV). Máscara de oxigênio a $100 \%(51 / \mathrm{min})$ foi colocada sobre a face da ovelha, para manter a saturação arterial de oxigênio em torno de $90 \%$ a $95 \%$.

Foram cateterizadas linhas venosas e arteriais, tanto na ovelha quanto no feto, e ambos os eletrocardiogramas e as pressões arteriais foram monitorizados num poligrafo de multicanais (Hewlett-Packard 7700, Hewlett-Packard Company, Palo Alto, CA). A anestesia fetal foi feita com injeção de ketamina (50 $\mathrm{mg} / \mathrm{kg}$ - I.M.) logo após a abertura da cavidade uterina.

\section{Procedimento}

A ovelha foi colocada em decúbito dorsal horizontal e preparada para intervenção cirúrgica estéril. O útero foi exposto através de laparotomia mediana infra-umbilical. $\mathrm{O}$ membro dianteiro direito do feto foi localizado através da palpação do útero. Após incisão de $10 \mathrm{~cm}$, o líqüido amniótico foi aspirado em condições estéreis e armazenado com gentamicina $(20 \mathrm{mg})$ a $37^{\circ} \mathrm{C}$. O membro foi, então, extraido para expor o hemitórax direito. A artéria braquial direita foi cateterizada para monitorizar a pressão arterial sistêmica e coletar sangue para análise de gases sangüíneos (Ciba-Corning 288; Medfield, MA). Em seguida, o coração fetal foi exposto através de toracotomia anterior direita $\left(6^{\circ}\right.$ ou $7^{\circ}$ espaço intercostal). O ECG basal foi obtido através de quatro eletrodos implantados na parede do toráx fetal, exteriorizados e fixados na pele materna. Foi realizada pericardiotomia para expor o átrio direito e ventrículo direito.

BAVT fetal e implante de marcapasso: O eletrodo de sutura epimiocárdica (Medtronic SP-5528, Medtronic Inc., Minneapolis, MN) foi implantado com 2 pontos de Prolene 7-0 no ventrículo direito de 6 fetos, e o eletrodo de rosqueamento modificado (Daig ML-151, Daig Corporation, Minnetonka, MN) foi implantado no ventrículo direito de outros 6 fetos (Figura 1).

As especificaçōes técnicas dos eletrodos estão na Tabela 1. Os eletrodos não continham esteróides. Após o implante, o eletrodo foi então conectado ao 
ASSAD, R. S.; JATENE, M. B.; MOREIRA, L. F. P.; SALES, P. C.; AIELLO, V. D.; COSTA, R.; HANLEY, F. L.; JATENE, A. D. -

Bloqueio AV total congênito: novo modelo experimental para avaliação do marcapasso fetal. Rev. Bras. Cir. Cardiovasc., 9 (3): 133-140, 1994

Animais de Laboratório", preparado pela Academia Nacional de Ciências e publicado pelo Instituto Nacional de Saúde dos E.U.A.

Estudos morfológicos: Os coraçōes fetais foram estudados após o óbito fetal ( 0 a 12 dias de pós-operatório). 0 órgão foi fixado logo após sua retirada, com formol tamponado a $10 \%$ por 24 horas. Um bloco de tecido abrangendo a área da junção atrioventricular, do seio coronário à crista supraventricular, foi ressecado e dividido em três partes. Foram também retiradas amostras da parede atrial e do local de implante do eletrodo no ventrículo direito. Os cortes histológicos foram obtidos com espessura de $5 \mu$, perpendicular ao anel AV. Dois cortes em 100 foram selecionados e corados com tricrômio de Masson e hematoxilina-eosina, respectivamente.

Análise estatística: Foi utilizado o Teste $t$ de Student para comparação dos dois eletrodos. A significância estatística foi estabelecida ao nível de $5 \%$. Todos os valores foram expressos como médias \pm erro padrão das médias (EPM).

\section{RESULTADOS}

O BAVT foi obtido em todos os animais. Apenas um feto reverteu espontaneamente para rítmo sinusal após 3 minutos em BAVT. Foi realizada nova crioablação do nó $A V$ e o bloqueio definitivo foi atingido. Não houve rítmo de escape ventricular após a indução do BAVT, e a freqüência atrial média foi de $165,0 \pm 8,4 \mathrm{bpm}$. O ECG fetal antes e após - BAVT está ilustrado na Figura 3.

Os gases arteriais e o hematócrito dos fetos antes e depois do procedimento permaneceram dentro dos limites fisiológicos dos ovinos ${ }^{3,12}$. Não houve alteraçōes hemodinâmicas decorrentes do procedimento em nenhum feto, com a pressão arterial fetal mantida nos mesmos niveis basais.

Achados histológicos: Consistiram em necrose parcial dos miócitos do nó AV, envolvendo o feixe de condução, as fibras adjacentes à crista, e a margem direita do septo interventricular. A lesão

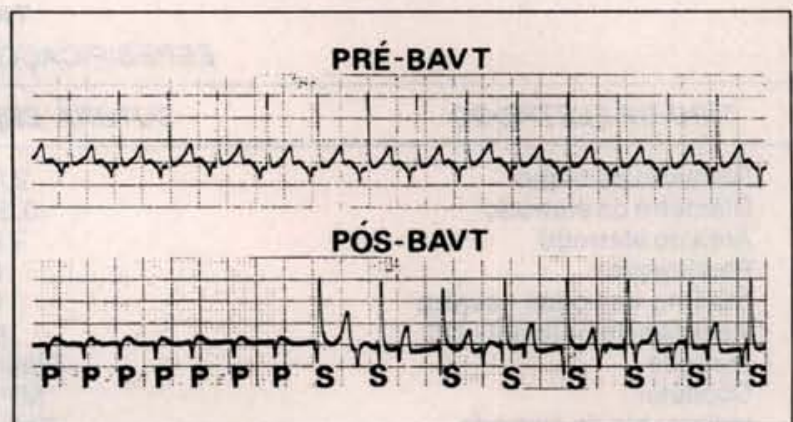

Fig. 3 - Superior: Eletrocardiograma basal do feto de ovelha \# 5 (ECG derivação II). Inferior: ECG do bloqueio AV total induzido pela crioablaçāo cirúrgica do nó AV. Observe a ausência de ritmo de escape ventricular.

$\mathrm{P}$ indica onda $\mathrm{P}, \mathrm{S}=$ estímulo, $\mathrm{BAVT}=$ bloqueio $\mathrm{AV}$ total.

poupou o ramo esquerdo e a porção distal do ramo direito.

Parâmetros dos eletrodos: O eletrodo de sutura epimiocárdica apresentou maiores dificuldades técnicas ao implante. Em ambos os grupos, não ocorreu estimulação do diafragma ou do nervo frênico.

Os valores obtidos para os parâmetros de sensibilidade antes do BAVT estão resumidos na Tabela 2. Não houve diferença estatisticamente significante entre os valores da onda $R$ de ambos os eletrodos, embora tenha ocorrido uma tendência para maior sensibilidade com o eletrodo de rosqueamento. Foi encontrado um slew rate menor $(0,62 \pm 0,2 \mathrm{~V} / \mathrm{seg})$ no grupo do eletrodo de sutura epimiocárdica, quando comparado ao eletrodo de rosqueamento $(1,40 \pm 0,2 \mathrm{~V} / \mathrm{seg})$. Esta diferença foi estatisticamente significante $(p=0,04)$.

As resistências dos 2 eletrodos não apresentaram uma diferença estatisticamente significante $476,3 \pm 29,8 \Omega$ para o de rosqueamento e $504,5 \pm$ $38,6 \Omega$ para $\circ$ de sutura epimiocárdica $(p=0,58)$. Os limiares de estimulação agudos foram constantemente baixos, sem falhas no estímulo dos dois eletrodos (Tabela 3 ). Os limiares de estimulaçāo agudos foram significantemente maiores para 0

TABELA 2

LIMIARES DE SENSIBILIDADE

\begin{tabular}{lcc}
\hline TIPO DE ELETRODO & ONDA R (MV) & SLEW RATE (VISEG) \\
\hline Sutura epimiocárdica & $10,3 \pm 1,4$ & $0,62 \pm 0,2$ \\
Rosqueamento & $14,7 \pm 2,5$ & $1,4 \pm 0,2$ \\
Valor de p & 0,218 & 0,042 \\
\hline
\end{tabular}


ASSAD, R. S.; JATENE, M. B.; MOREIRA, L. F. P.; SALES, P. C.; AIELLO, V. D.; COSTA, R.; HANLEY, F. L.; JATENE, A. D. Bloqueio AV total congênito: novo modelo experimental para avaliação do marcapasso fetal. Rev. Bras. Cir. Cardiovasc., 9 (3): 133-140, 1994

TABELA 1

ESPECIFICAÇÕES DOS ELETRODOS

\begin{tabular}{lcc}
\hline TIPO DE ELETRODO & SUTURA EPIMIOCÁRDICA & ROSQUEAMENTO \\
\hline Diâmetro do corpo & $2,0 \mathrm{~mm}$ & $1,8 \mathrm{~mm}$ \\
Diâmetro do eletrodo & $0,56 \mathrm{~mm}$ & $4,2 \mathrm{~mm}($ espira) \\
Área do eletrodo & $7 \mathrm{~mm} 2$ & $10 \mathrm{~mm}^{2}$ \\
Penetração & $\mathrm{NA}$ & $2,0 \mathrm{~mm}$ \\
Número de voltas - espira & $\mathrm{NA}$ & 1,5 \\
Resistência nominal - DC & $80 \Omega$ & $27 \Omega$ \\
Eletrodo & Platina/irídio & Platina/irídio \\
Condutor & MP - 35N & Niquel "alloy" \\
Isolamento do eletrodo & Poliuretano & Borracha siliconizada \\
Pino conector & Aço inoxidável inoxidável \\
\hline
\end{tabular}

NA = Não aplicável

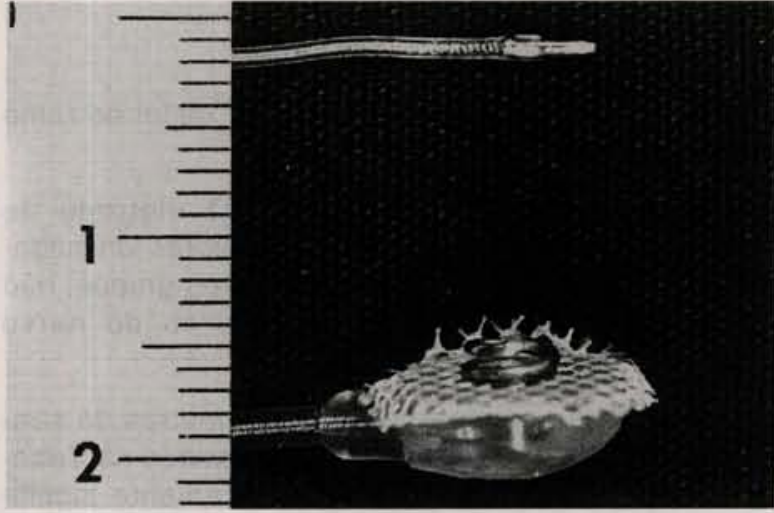

Fig. 1- Eletrodo de sutura epimiocárdica Medtronic SP-5528, com uma área de $7 \mathrm{~mm}^{2}$ (acima) e o eletrodo de rosqueamento modificado, com 1,5 espiras e uma área de $10 \mathrm{~mm}^{2}$ (abaixo).

analisador de pulsos (Medtronic 5311-B) para medir - limiar de sensibilidade (onda $\mathrm{R}$ e slew rate) antes de induzir o bloqueio. Com monitorização eletrocardiográfica contínua, o BAVT fetal foi obtido através da crioablação cirúrgica do nó AV, sem suporte de circulação extracorpórea. A ponteira do crioablador com $3 \mathrm{~mm}$ de diâmetro (Figitronics Cryosurgical System CCS 100, Shelton, CT) foi aplicada sobre o seio coronário, comprimindo a parede atrial contra 0 anel da valva tricúspide, conforme ilustrado na Figura 2. A área foi resfriada para $-60^{\circ} \mathrm{C}$ durante 30 segundos e logo após reaquecida. O BAVT fetal foi confirmado por inspeção visual da dissociação AV e gravação contínua do ECG. Quando o BAVT foi obtido, o marcapasso ventricular foi iniciado com uma freqüência de $120 \mathrm{bpm}$.

Limiar de estimulação do miocárdio fetal: As medidas foram feitas com largura de pulso constante (Medtronic 5311-B), até que a menor voltagem proporcionasse $100 \%$ de captura ventricular. Os limiares de estimulação foram medidos com largura de pulso de $0,1 \mathrm{mseg}$ a $2,0 \mathrm{mseg}$, através da dimi-

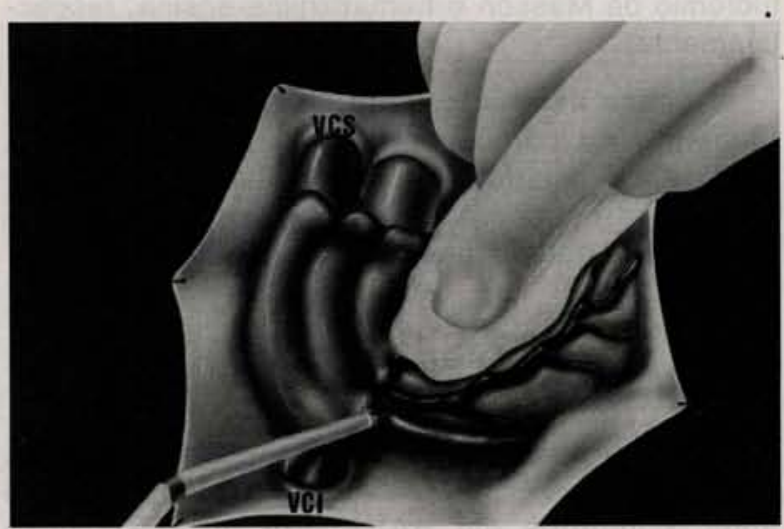

Fig. 2 - Detalhe da crioablação cirúrgica do nó AV sobre o seio coronário, com uma ponteira de congelamento de $3 \mathrm{~mm}$ de diâmetro. VCS = veia cava superior; $\mathrm{VCI}=$ veia cava inferior; $\mathrm{SC}=$ seio coronário.

nuição gradual da voltagem do analisador de pulso até a ocorrência da assistolia. A corrente para aquele limiar específico foi, então, medida. A resistência foi medida com voltagem constante $(5 \mathrm{~V})$ e $0,5 \mathrm{mseg}$ de largura de pulso.

Após todas as medidas, o eletrodo foi conectado ao marcapasso (Dialog II, Siemens-Elema AB, Solna, Suécia), que ficou alojado na parede torácica fetal com gentamicina local $(20 \mathrm{mg})$. O marcapasso ficou regulado com os seguintes parâmetros: modo VVI, freqüência de 120 pulsos/min, sensibilidade 1,25 $\mathrm{mV}$, amplitude de pulso $5,0 \mathrm{~V}$, e período refratário 400 mseg.

A toracotomia fetal e o útero foram fechados com sutura contínua de fio absorvível. O líqüido amniótico armazenado foi devolvido à cavidade amniótica antes do final da sutura uterina. Os fetos foram para sobrevida para posterior estudo microscópico da área do nó AV.

Todos os animais receberam tratamento humano de acordo com o "Guia de Cuidados e Uso de 
ASSAD, R. S.; JATENE, M. B.; MOREIRA, L. F. P.; SALES, P. C.; AIELLO, V. D.; COSTA, R.; HANLEY, F. L.; JATENE, A. D. Bloqueio AV total congênito: novo modelo experimental para avaliação do marcapasso fetal. Rev. Bras. Cir. Cardiovasc., 9 (3): 133-140, 1994.

TABELA 3

PARÂMETROS DE ESTIMULAÇĀO - VOLTAGEM

\begin{tabular}{cccc}
\hline LARGURA DE PULSO (MSEG) & \multicolumn{2}{c}{ VOLTAGEM(V) } & VALOR DE P \\
\cline { 2 - 4 } & SUTURAEPICARDICA & ROSQUEAMENTO & \\
\hline 0,1 & $3,02 \pm 0,4$ & $1,43 \pm 0,4$ & 0,013 \\
0,2 & $1,72 \pm 0,2$ & $0,88 \pm 0,2$ & 0,016 \\
0,3 & $1,33 \pm 0,1$ & $0,73 \pm 0,2$ & 0,015 \\
0,4 & $1,08 \pm 0,1$ & $0,62 \pm 0,1$ & 0,022 \\
0,5 & $0,97 \pm 0,1$ & $0,53 \pm 0,1$ & 0,018 \\
0,6 & $0,85 \pm 0,1$ & $0,50 \pm 0,1$ & 0,013 \\
0,7 & $0,78 \pm 0,1$ & $0,45 \pm 0,1$ & 0,011 \\
0,8 & $0,77 \pm 0,1$ & $0,43 \pm 0,1$ & 0,006 \\
0,9 & $0,68 \pm 0,1$ & $0,42 \pm 0,1$ & 0,018 \\
1,0 & $0,65 \pm 0,1$ & $0,42 \pm 0,1$ & 0,056 \\
1,5 & $0,60 \pm 0,1$ & $0,40 \pm 0,1$ & 0,028 \\
2,0 & $0,57 \pm 0,1$ & $0,40 \pm 0,1$ & 0,102 \\
\hline
\end{tabular}

eletrodo de sutura epimiocárdica com largura de pulso abaixo de $0,9 \mathrm{mseg}$.

A Figura 4 mostra a curva de voltagem $x$ largura de pulso para os limiares agudos de estimulação de ambos os eletrodos. As duas curvas ${ }^{7}$ permaneceram relativamente constantes com valores de largura de pulso maiores que $0,6 \mathrm{mseg}$, subindo agudamente com valores menores de largura de pulso em ambos os eletrodos. Por outro lado, a corrente medida no limiar de voltagem com largura de pulso menor que $0,5 \mathrm{mseg}$ foi significativamente menor com o eletrodo de rosqueamento (Tabela 4).

\section{COMENTÁRIOS}

A técnica de crioablação cirúrgica do nó AV descrita proporciona um método alternativo de se induzir o BAVT no feto, sem necessidade de circu-

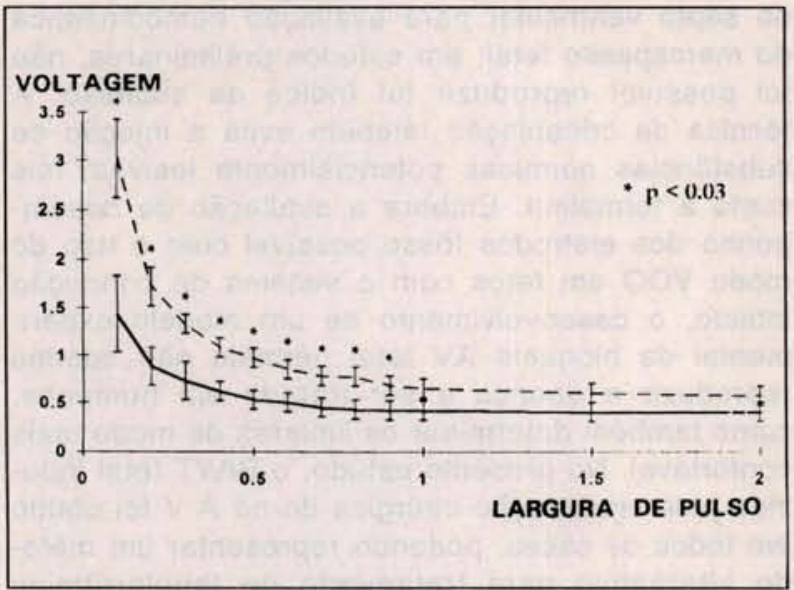

Fig. 4 - Curvas de voltagem $\times$ largura de pulso (média \pm EPM) para o limiar agudo de estimulação do miocárdico fetal. Linha cheia=eletrodo de rosqueamento; linha pontilhada=eletrodo de sutura epimiocárdica.

"p $<0,03$

TABELA 4

PARÂMETROS DE ESTIMULAÇÃO - CORRENTE

\begin{tabular}{cccc}
\hline LARGURA DE PULSO (MSEG) & \multicolumn{2}{c}{ CORRENTE (MA) } & VALOR DE P \\
\cline { 2 - 3 } & SUTURAEPIMIOCARDICA & ROSQUEAMENTO \\
\hline 0,1 & $6,08 \pm 0,4$ & $3,28 \pm 0,8$ & 0,048 \\
0,2 & $3,60 \pm 0,2$ & $1,92 \pm 0,5$ & 0,044 \\
0,3 & $2,80 \pm 0,2$ & $1,57 \pm 0,3$ & 0,028 \\
0,4 & $2,35 \pm 0,1$ & $1,37 \pm 0,3$ & 0,05 \\
0,5 & $2,08 \pm 0,1$ & $1,20 \pm 0,3$ & 0,047 \\
0,6 & $1,83 \pm 0,1$ & $1,13 \pm 0,2$ & 0,067 \\
0,7 & $1,67 \pm 0,1$ & $1,02 \pm 0,2$ & 0,059 \\
0,8 & $1,67 \pm 0,1$ & $0,95 \pm 0,2$ & 0,029 \\
0,9 & $1,53 \pm 0,1$ & $0,97 \pm 0,2$ & 0,062 \\
1,0 & $1,47 \pm 0,1$ & $0,95 \pm 0,2$ & 0,096 \\
1,5 & $1,40 \pm 0,1$ & $0,90 \pm 0,1$ & 0,053 \\
2,0 & $1,27 \pm 0,1$ & $0,87 \pm 0,1$ & 0,136 \\
\hline
\end{tabular}


ASSAD, R. S.; JATENE, M. B.; MOREIRA, L. F. P.; SALES, P. C.; AIELLO, V. D.; COSTA, R.; HANLEY, F. L.; JATENE, A. D. Bloqueio AV total congênito: novo modelo experimental para avaliação do marcapasso fetal. Rev. Bras. Cir. Cardiovasc., 9 (3): $133-140,1994$.

lação extracorpórea e/ou intervenção vascular. Uma característica importante das lesões criogênicas é a resistência marcante dos elementos vasculares, colágeno, e fibroblastos à lesão hipotérmica, o que pode explicar a ausência de rotura ou formação de aneurismas nas lesões após o congelamento 7,25 . A manutenção do equilíbrio hemodinâmico após o BAVT pode estar relacionada à ausência de infarto do miocárdio septal e ao padrão focal da lesão de crioablação sobre o nó AV.

Até o presente, as informações de pesquisas em circulação extracorpórea fetal não permitem uma abordagem transatrial segura. Além do mais, a manipulação do frágil tecido atrial fetal é evitada com esta técnica. Nossos resultados comparam favoravelmente com os resultados descritos por CROMBLEHOLME et alii ${ }^{8}$, que obtiveram BAVT em $90 \%$ dos fetos ovinos através da injeção de formalina no septo ventricular para avaliação hemodinâmica do marcapasso fetal; em estudos preliminares, não foi possivel reproduzir tal índice de sucesso. A técnica da crioablação também evita a injeção de substâncias químicas potencialmente lesivas, tais como a formalina. Embora a avaliação do desempenho dos eletrodos fosse possível com o uso do modo VOO em fetos com o sistema de condução intacto, o desenvolvimento de um modelo experimental de bloqueio AV fetal permitiu não apenas reproduzir a doença a ser tratada em humanos, como também determinar os limiares de modo mais confortável. No presente estudo, o BAVT fetal induzido pela crioablação cirúrgica do nó $\mathrm{A} V$ foi obtido em todos os casos, podendo representar um método alternativo para tratamento de taquiarritmias supraventriculares fetais refratárias ao tratamento clínico.

Os eletrodos epicárdicos e intravasculares são meios bem estabelecidos de condução do estímulo do gerador para o coração 19,26 . Entretanto, acreditamos que o uso do eletrodo epicárdico para implante de marcapasso em fetos proporciona uma fixação mais estável com desempenho aceitável. Estudos de HEINEMANN et alii ${ }^{13}$ e SCAGLIOTTI et alii ${ }^{21}$ demonstraram a possibilidade do implante de marcapasso intra-uterino em fetos de ovelhas, usando eletrodos epicárdicos. Nosso estudo avaliou dois eletrodos epicárdicos distintos, através de um novo modelo de BAVT em fetos de ovinos. Ambos os eletrodos apresentaram baixos limiares agudos e os resultados com o eletrodo de rosqueamento foram superiores aos valores obtidos com os eletrodos de sutura epimiocárdica. O limiar de estimulação durante o implante foi menor com o eletrodo de rosqueamento para valores mais baixos de largura de pulso, proporcionando melhor conservação de energia. Um aumento maior do limiar pode ocorrer com este eletrodo nas primeiras 4 a 6 semanas após o implante, quando comparado ao eletrodo de sutura epimiocárdica. Atualmente, pesquisas contínuas da avaliação tardia da crioablação do nó AV fetal proposta neste estudo e dos limiares crônicos dos eletrodos estão sendo prosseguidas em nossos laboratórios.

A resistência de estimulação discretamente maior encontrada nos eletrodos de sutura epimiocárdica pode ser devido ao contato direto do tecido com menos da metade da superfície do eletrodo. Embora o analisador de pulsos tenha acusado uma onda $\mathrm{R}$ adequada para ambos os eletrodos (maior que $10 \mathrm{mV}$ ), a probabilidade de perda de captura é elevada com os eletrodos de sutura epimiocárdica (slew rate < 1,0 V/seg durante o implante) 4, 11. Nesse caso, FURMAN et alii ${ }^{11}$ recomendam o uso de gerador de pulsos de alta sensibilidade ou com sensibilidade programável para optimizar a sensibilidade do gerador.

Concluímos que o implante de marcapasso epicárdico em fetos com eletrodo de rosqueamento, via toracotomia, é factível e reprodutivel, apresentando melhor desempenho e consideráveis vantagens técnicas. Entretanto, este procedimento deve ser baseado em análise crítica da relação de risco/ benefício, considerando-se o risco cirúrgico materno tanto como a recuperação potencial do feto, com BAVT e hidropsia. O trabalho de parto prematuro ainda representa um desafio para a cirurgia fetal ${ }^{17}$. Avanços nas pesquisas da resposta fetal ao estresse cirúrgico e controle farmacológico do parto prematuro após a intervenção intra-uterina podem fazer com que esta alternativa terapêutica seja aplicável em humanos num futuro próximo. 
ASSAD, R. S.; JATENE, M. B.; MOREIRA, L. F. P.; SALES, P. C.; AIELLO, V. D.; COSTA, R.; HANLEY, F. L.; JATENE, A. D. - Fetal heart block: a new experimental model to assess fetal pacing. Rev. Bras. Cir. Cardiovasc., 9 (3): 133-140, 1994.

ABSTRACT: Epicardial fetal pacing via thoracotomy has the potential for being a safer and more reliable procedure to treat congenital complete heart block (CHB) associated with fetal hydrops refractory to medical therapy. To assess the acute electrophysiologic characteristics of two ventricular epicardial leads, a new experimental model of fetal heart block induced by cryosurgical ablation of the AV node without the need for fetal cardiac bypass was performed in 12 pregnant ewes at 110-115 days of gestation. A modified screw-in lead (one and a half turn) was used in 6 fetal lambs and a stitch-on lead in the other 6 lambs. CHB was achieved in $100 \%$ of the fetal lambs, with no ventricular escape rate noticed in any of the lambs. The acute stimulation thresholds were consistently low for both the leads, with lower values for the screw-in lead at pulse duration below $0.9 \mathrm{~ms}(p<0.03)$. Current measured at voltage threshold with pulse width below $0.5 \mathrm{~ms}$ was lower for the screw-in lead $(p<0.048)$. Stimulation resistance, measured during constant-voltage pacing, was not statistically different between the two leads $(441.8 \pm 13.7 \Omega$ for the screw-in lead versus $480.2 \pm 59.2 \Omega$ for the stitch-on lead). No significant differences $(p>0.20)$ were found in R-wave amplitude between the two electrodes. Slew rates were significantly higher with the screw-in group than with the stitch-on group $(1.40 \pm$ 0.2 versus $0.62 \pm 0.2 \mathrm{~V} / \mathrm{s}, \mathrm{p}=0.04)$. This model of $\mathrm{CHB}$ is a simple and reproducible method to assess fetal pacing. We find the screw-in electrode a better option when fetal pacing is indicated.

DESCRIPTORS: fetal cardiology; fetal heart block; fetal surgery; fetal arrhythmias; fetal pacing.

AGRADECIMENTO: Agradecemos o apoio prestado pelos Drs. Sony D. Bicudo, da Faculdade de Medicina Veterinária e Zooctecnia, UNESP - Campus de Botucatu, SP e José A. Visintin, da Faculdade de Medicina Veterinária e Zootecnia da Universidade de São Paulo.

\section{REFERÊNCIAS BIBLIOGRÁFICAS}

1 ALLAN, L. D.; ANDERSON, R. H.; SULLIVAN, I. D.; CAMPBELL, S.; HOLT, D. W.; TYNAN, M. Evaluation of fetal arrhythmias by echocardiography. Br. Heart J., 50: 240-245, 1983.

2 ALLAN, L. D.; CRAWFORD, D. C.; ANDERSON, R. H.; TYNAN, M. - Evaluation and treatment of fetal arrhythmias. Clin. Cardiol., 7: 467-473, 1984.

3 ASSALI, N. S.; BRINKMAN, C. R.; NUWAYHID, B. Comparison of maternal and fetal cardiovascular functions in acute and chronic experiments in the sheep. Am. J. Obstet. Gynecol., 120: 411-425, 1974.

BAROLD, S. S.; ONG, L. S.; HEINLE, R. A. - Stimulation and sensing thresholds for cardiac pacing: electrophysiologic and technical aspects. Progr. Cardiovasc. Dis., 24:1-24, 1981.
5 BIERMAN, F. Z.; BAXI, L.; JAFFE, I.; DRISCOLL, J. Fetal hydrops and congenital complete heart block: response to maternal steroid therapy. J Pediatr., 112: $646-648,1988$.

6 CARPENTER, Jr. R. J.; STRASBURGER, J. F.; GARSON Jr., A.; SMITH, R. T.; DETER, R. L.; ENGELHARDT Jr., H. T. - Fetal ventricular pacing for hydrops secondary to complete atrioventricular block. J. Am. Coll. Cardiol., 8: 1434-1436, 1986.

7 COOPER, I. S.; SAMRA, K.; WISNIEWSKA, K. - Effects of freezing on major arteries. Stroke, 2: 471-482, 1971.

8 CROMBLEHOLME, T. M.; HARRISON, M. R.; LONGAKER, M. T.; LANGER, J. C.; ADZICK, N. S.; BRADLEY, S.; DUNCAN, B.; VERRIER, E. D. Complete heart block in fetal lambs. I: Technique and acute physiological response. J. Pediatr. Surg., 25: 587-593, 1990.

9 CROWLEY, D. C.; DICK, M.; RAYBURN, W. F.; ROSENTHAL, A. - Two dimensional and M-mode echocardiographic evaluation of fetal arrhythmias. Clin. Cardiol., 8: 1-10, 1985.

10 DEVORE, G. R.; SIASSI, B.; PLATT, L. D. - Fetal echocardiography. III: The diagnosis of cardiac arrhythmias using real time directed $\mathrm{M}$-mode ultrasound. Am. J. Obstet. Gynecol., 146: 792-799, 1983. 
ASSAD, R. S.; JATENE, M. B.; MOREIRA, L. F. P.; SALES, P. C.; AIELLO, V. D.; COSTA, R.; HANLEY, F. L.; JATENE, A. D. Bloqueio AV total congênito: novo modelo experimental para avaliação do marcapasso fetal. Rev. Bras. Cir. Cardiovasc., 9 (3): $133-140,1994$

FURMAN, S.; HURZELER, P.; DeCAPRIO, V. - The ventricular endocardial electrogram and pacemaker sensing. J. Thorac. Cardiovasc. Surg., 73: 258-266, 1977.

12 GOETZMAN, B. W.; ITSKOVITZ, J.; RUDOLPH, A. M. - Fetal adaptations to spontaneous hypoxemia and responses to maternal oxygen breathing. Biol. Neonate, 46: 276-284, 1984.

13 HEINEMANN, M. K.; SABIK, J. F.; GAMBLE, W. J.; HANLEY, F. L. - Prenatal pacemaker implantation in the fetal lamb. Circulation, 84 (2): II-513, 1991 (Resumo).

14 HUHTA, J. C.; STRASBURGER, J. F.; CARPENTER, R. J. - Pulsed doppler fetal echocardiography. J. Clin. Ultrasound, 13: 247-254, 1985.

15 KLEINMAN, C. S.; DONNERSTEIN, R. L.; JAFFE, C. C.; DeVORE, G. R.; WEINSTEIN, E. M.; LYNCH, D. C.; TALNER, N. S.; BERKOWITZ, R. L.; HOBBINS, J. C. - Fetal echocardiography. A tool for evaluation of in utero cardiac arrhythmias and monitoring of in utero therapy: analysis of 71 patients. Am. J. Cardiol., 51: 237-243, 1983.

16 KLEINMAN, C. S.; HOBBINS, J. C.; JAFFE, C. C.; LYNCH, D. C.; TALNER, N. S. - Echocardiographic studies of the human fetus: prenatal diagnosis of congenital heart disease and cardiac dysrhythmias. Pediatrics. 65: 1059-1066, 1980.

17 LONGAKER, M. T.; GOLBUS, M. S.; FILLY, R. A.; ROSEN, M. A.; CHANG, S. A.; HARRISON, M. R. - Maternal outcome after open fetal surgery: a review of the first 17 human cases. JAMA, 265: 737-741, 1991.

18 MACHADO, M. V. L.; TYNAN, M. J.; CURRY, P. V. L.; ALLAN, L. D. - Fetal complete heart block. Br. Heart J., 60: 512-515, 1988.

19

MAGILLIGAN Jr., D. J.; HAKIMI, M.; DAVILA, J. C. The sutureless electrode: comparison with transvenous and sutured epicardial electrode

placement for permanent pacing. Ann. Thorac. Surg., 22: 80-86, 1976.

3

SCHIMIDT, K. G.; ULMER, H. E.; SILVERMAN, N. H.; KLEINMAN, C. S.; COPEL, J. A. - Perinatal outcome of fetal complete atrioventricular block: a multicenter experience. J. Am. Coll. Cardiol., 17: 1360-1366, 1991.

SCHULZ, D. M.; GIORDANO, D. A.; SHULZ, D. H. Weighs of organs of fetuses and infants. Arch. Pathol., 74: 244-250, 1962.

TAYLOR, C. B.; DAVIS, C. B.; VAWTER, G. F.; HASS, G. M. - Controlled myocardial injury produced by a hypothermal method. Circulation, 3: 239-253, 1951.

WALLS, J. T.; MALONEY, J. D.; PLUTH, J. R. - Clinical evaluation of a sutureless cardiac pacing lead: chronic threshold changes and lead durability. Ann. Thorac. Surg., 36: 328-331, 1983.

27 WLADIMIROFF, J. W. \& McGHIE, J. S. - Ultrasonic assessment of cardiovascular geometry and function in the human fetus. Br. J. Obstet. Gynecol., 88: $870-875,1981$

28 WLADIMIROFF, J. W.; VOSTERS, R.; McGHIE, J. S. Normal cardiac ventricular geometry and function during the last trimester of pregnancy and early neonatal period. Br. J. Obstet. Gynecol., 89: 839$844,1982$. 